\title{
Human Motor Plasticity Induced by Mirror Visual Feedback
}

\author{
Ippei Nojima, ${ }^{1,2}$ Tatsuya Mima, ${ }^{2}$ Satoko Koganemaru, ${ }^{2}$ Mohamed Nasreldin Thabit, ${ }^{2,3}$ Hidenao Fukuyama, ${ }^{2}$ \\ and Toshio Kawamata ${ }^{1}$ \\ ${ }^{1}$ Kobe University Graduate School of Health Science, Hyogo 654-0142, Japan, ${ }^{2}$ Human Brain Research Center, Kyoto University Graduate School of \\ Medicine, Kyoto 606-8507, Japan, and 3 Department of Neurology, Faculty of Medicine, Sohag University, Sohag 82524, Egypt
}

The clinical use of mirror visual feedback (MVF) was initially introduced to alleviate phantom pain, and has since been applied to the improvement of hemiparesis following stroke. However, it is not known whether MVF can restore motor function by producing plastic changes in the human primary motor cortex (M1). Here, we used transcranial magnetic stimulation to test whether M1 plasticity is a physiological substrate of MVF-induced motor behavioral improvement. MVF intervention in normal volunteers using a mirror box improved motor behavior and enhanced excitatory functions of the M1. Moreover, behavioral and physiological measures of MVFinduced changes were positively correlated with each other. Improved motor performance occurred after observation of a simple action, but not after repetitive motor training of the nontarget hand without MVF, suggesting the crucial importance of visual feedback. The beneficial effects of MVF were disrupted by continuous theta burst stimulation (cTBS) over the M1, but not the control site in the occipital cortex. However, MVF following cTBS could further improve the motor functions. Our findings indicate that M1 plasticity, especially in its excitatory connections, is an essential component of MVF-based therapies.

\section{Introduction}

Mirror therapy is a promising novel intervention technique that was recently introduced for the rehabilitation of patients with hemiparesis. It was originally used to treat phantom limb pain in amputees (Ramachandran and Rogers-Ramachandran, 1996) by superimposing a visual image of the intact limb on the phantom one using mirror visual feedback (MVF). This technique has also been reported to be useful in patients with complex regional pain syndrome (McCabe et al., 2003; Moseley, 2004), and has been applied to stroke patients with motor deficits, in whom motor training of the unimpaired limb with its MVF superimposed over the paretic limb led to a remarkable motor recovery (Altschuler et al., 1999; Sathian et al., 2000; Stevens and Stoykov, 2003; Sütbeyaz et al., 2007; Yavuzer et al., 2008). However, the physiological mechanism of MVF-induced beneficial effects is not well understood.

Several previous studies have suggested that MVF can increase primary motor cortex (M1) activity of the stationary hand. An MEG study showed that the human left M1 was strongly activated by a visual image of the moving hand when the subjects watched both the right hand and the MVF of the left hand (Tominaga et al., 2009). However, the oscillatory activity of the sensorimotor cortex is an indirect measure of M1 excitability, because its neurobiological meaning has not been fully clarified (Oishi et al.,

\footnotetext{
Received Oct. 24, 2011; revised Nov. 30, 2011; accepted Dec. 6, 2011

Author contributions: I.N. and T.M. designed research; I.N. and M.N.T. performed research; I.N., S.K., H.F., and T.K. contributed unpublished reagents/analytic tools; I.N. analyzed data; I.N. wrote the paper.

This study was supported by Grant-in-Aid for Scientific Research (C) 21613003 (to T.M.) from the Japan Society for the Promotion of Science.

Correspondence should be addressed to Dr. Tatsuya Mima, Department of Brain Pathophysiology, Human Brain Research Center, Kyoto University Graduate School of Medicine, Shogoin Kawahara-cho 54, Sakyo-ku, Kyoto 6068507, Japan. E-mail:mima@kuhp.kyoto-u.ac.jp.

DOI:10.1523/JNEUROSCI.5364-11.2012

Copyright $\odot 2012$ the authors $\quad 0270-6474 / 12 / 321293-08 \$ 15.00 / 0$
}

2007). Another study using transcranial magnetic stimulation (TMS) revealed an increase in M1 excitability of the stationary hand during MVF (Garry et al., 2005), and a functional magnetic resonance imaging ( $\mathrm{fMRI}$ ) study recorded increased activation in the M1 of the affected limb of brachial plexus avulsion patients following an 8 week MVF training program using a virtual reality system (Giraux and Sirigu, 2003).

It is not yet known whether the MVF exerts its positive effects in motor behavior by influencing motor plasticity, so here we used TMS to investigate the plastic changes of the M1. We hypothesized that motor training of the hand would produce a plastic change in motor behavior and M1 function of the contralateral hand when MVF was applied to the subject. Using TMS allowed us to study the excitatory and inhibitory functions of the human M1 extensively and noninvasively (Pascual-Leone et al., 1998; Koganemaru et al., 2009, 2010; Thabit et al., 2010). In addition, we tested whether continuous theta burst stimulation (cTBS) over the M1 (Huang and Rothwell, 2004; Huang et al., 2005) can disrupt the behavioral and electrophysiological motor improvement induced by MVF.

\section{Materials and Methods}

Subjects. Sixty-three neurologically healthy subjects (34 males and 29 females; age, $24.7 \pm 4.7$ years, mean $\pm \mathrm{SD}$ ) participated in the study. None of the participants had a history of neurological illness or was taking medication. All volunteers were right-handed according to the Oldfield handedness inventory (Oldfield, 1971). The study was approved by the Ethics Committee of the Kyoto University Graduate School and Faculty of Medicine (Kyoto, Japan). All subjects gave written informed consent before their participation.

Recording procedures. Electromyograms were recorded from the right and left first dorsal interosseous (FDI) and flexor carpi radialis muscles using surface silver/silver chloride $(\mathrm{Ag} / \mathrm{AgCl})$ electrodes. The electromyograms were amplified and filtered (bandpass, 5-2000 Hz), and digitized at a sampling rate of $10 \mathrm{kHz}$ using the Neuroscan system. 
TMS was performed with two Magstim 200 magnetic stimulators connected by a bistim module. This device allows delivery of two magnetic stimulations through the same coil. The handle of the coil pointed backwards and $45^{\circ}$ lateral to the midline. A single pulse of TMS was delivered using a flat figure-of-eight magnetic coil (outer diameter of each wing, 9 $\mathrm{cm}$ ) at the optimal scalp positions to induce a motor response for the left FDI muscle.

The motor threshold (MT) was determined for both relaxed (rMT) and tonic active (aMT) muscles as the minimum stimulation intensity (\%) that evoked a clearly distinguishable motor-evoked potential (MEP). The rMT was defined as the lowest stimulus intensity sufficient to elicit five MEPs of $\geq 50 \mu \mathrm{V}$ in a series of 10 stimuli delivered with at least $5 \mathrm{~s}$ intervals (Rossini et al., 1994). In determining the aMT, subjects were instructed to maintain a steady muscle contraction of $30 \%$ of their maximum voluntary contraction. The aMT was defined as the lowest stimulus intensity sufficient to elicit MEPs with an amplitude of $\sim 200 \mu \mathrm{V}$ that could be distinguished from the background electromyography signal in $50 \%$ of trials (Rothwell et al., 1999).

To assess corticospinal excitability, MEP amplitudes were measured with the fixed stimulus intensity of the TMS machine adjusted to produce an MEP of $\sim 1 \mathrm{mV}$ from the target FDI muscle before the intervention $\left(\mathrm{SI}_{1 \mathrm{mV}}\right)$.

The recruitment of the corticospinal projection (I-O function) from the right M1 was also measured (Ridding and Rothwell, 1997). The intensities of single TMS stimuli were individually adapted according to the rMT to evaluate the I-O function. Eight MEPs were recorded from the left FDI muscle at intensities of 50, 80, 90, 100, 110, 120, 130, and 150\% of the rMT.

The short-latency intracortical inhibition (SICI) and interhemispheric inhibition (IHI) were measured to assess the inhibitory system. SICI was measured according to a paired conditioning test stimulus paradigm with a subthreshold conditioning stimulus at $95 \%$ of the aMT followed by a suprathreshold test stimulus at $\mathrm{SI}_{1 \mathrm{mV}}$ with an interstimulus interval (ISI) of 3 ms (Kujirai et al., 1993; Ziemann et al., 1996b). The protocol used to measure IHI was similar to that described previously (Ferbert et al., 1992). The conditioning stimulus at $\mathrm{SI}_{1 \mathrm{mV}}$ was applied to the left $\mathrm{M} 1$ and the test stimulus at $\mathrm{SI}_{1 \mathrm{mV}}$ was applied to the right M1 10 or $40 \mathrm{~ms}$ later (Chen et al., 2003). The size of the mean conditioned response for SICI, IHI(10), and IHI(40) (10 trials each) was expressed as a percentage of the size of the mean test response alone.

With the exception of the aMT, both hands were at rest and muscle relaxation was visually monitored by the electromyographic signal.

F-wave. Supramaximal electrical stimulation of the ulnar nerve at the wrist was performed in a separate session to measure the F-wave from the left FDI to test the effects of mirror therapy at the spinal level in six subjects (Mercuri et al., 1996). Sixteen F-waves were recorded and averaged before and after MVF intervention.

Ball-rotation task. Subjects were seated in a comfortable chair with their elbows flexed at $90^{\circ}$ and their hands pronated in a totally relaxed position. The target task was to rotate two cork balls (diameter, $30 \mathrm{~mm}$; weight, $10 \mathrm{~g}$ ) as fast as possible in a counterclockwise direction with the left hand. The dexterity of the motor performance of the left hand was examined by counting the number of two-ball rotations during $30 \mathrm{~s}$. After the experiments, one of the experimenters, who was blind to the participants' status, reviewed the video recording and counted the number of rotations.

Experiment 1: mirror versus nonmirror tasks. To investigate the effect of MVF on M1 plasticity, we tested the change in motor function of the left hand both behaviorally and electrophysiologically, after motor training of the right hand with and without MVF (mirror vs nonmirror task). Twenty healthy subjects ( 16 males and four females) were assigned to this experiment.

Motor-training intervention. Subjects were randomly assigned to the mirror task group $(n=10)$ or the nonmirror task group $(n=10)$. For the mirror task, subjects placed both hands inside a box made of wood and mirrored glass. In this and other interventions, they could not see the right hand directly. They practiced 10 sets of the clockwise rotation of two balls for $30 \mathrm{~s}$ each using the right hand with MVF superimposed on the left hand. Subjects were asked to relax their left hand, which was continuously monitored and confirmed by surface EMG. Care was taken to ensure that the mirror image of the right hand was shown over the real left hand. Subjects practiced by completing 10 sets of two-ball rotations for $30 \mathrm{~s}$ with a $30 \mathrm{~s}$ rest interval to avoid fatigue (total of $270 \mathrm{~s}$ ). For the nonmirror task, subjects placed both hands inside a box made of wood and transparent glass. They performed the same motor training of the right hand as that performed by the mirror task group, but MVF was not applied. To assess the motor function of the left hand before and after the motor-training intervention, the dexterity of the left hand (the number of ball rotations in $30 \mathrm{~s}$ ) and various TMS parameters were evaluated (Pre, Post; Fig. 1, top).

Experiment 2: mirror versus action observation tasks. We investigated the action observation (AO) task to test whether MVF-induced motor improvement of the left hand is caused by repetitive training of the right hand or visual feedback of the left-hand movement. The glass of the mirror box was replaced by an LCD monitor, and the relaxed subjects observed a recording of another person carrying out motor-training using the inverted right hand $(270 \mathrm{~s})$, while not making any movement themselves. Care was taken to ensure that the on-screen hand was approximately the same size as the subject's hand. Subjects were asked to concentrate on the video with both of their hands relaxed. Subjects were asked not to actively imagine motor training of their own left hand to avoid possible interference of motor imagery on motor learning effects. Twenty-seven healthy subjects ( 12 males and 15 females) were assigned to this experiment (nine subjects each for the mirror, nonmirror, and $\mathrm{AO}$ tasks).

To assess the motor function of the left and right hands before and after the motor-training intervention, the dexterity of both (that is, the number of ball rotations in $30 \mathrm{~s}$ ) was evaluated (Pre, Post; Fig. 1, top).

Experiment 3: effects of cTBS. To confirm the functional relevance of the M1 in the motor improvement induced by MVF, we used cTBS to provide functional interference over the right $\mathrm{M} 1$ or occipital area (OC) just after MVF intervention (Fig. 1, bottom). Sixteen healthy subjects (six males and 10 females) who did not participate in Experiments 1 or 2 were assigned to this trial.

Subjects were randomly assigned to the M1 group $(n=8)$ or the OC group $(n=8)$ according to the site at which cTBS was applied. Motor functions of the left hand were measured by hand dexterity as before, and MEP amplitude of the left FDI at $\mathrm{SI}_{1 \mathrm{mV}}$. After measuring the motor functions (Pre), subjects practiced motor-training intervention of the right hand with MVF superimposed on the left hand using a mirror box (mirror task). As in Experiment 1, motor functions were then remeasured (Post1). cTBS was applied over either the right $\mathrm{M} 1$ or the $\mathrm{OC}$, and the motor functions were measured again (Post2). For cTBS, TMS was delivered as 200 bursts of three pulses (ISI $=20 \mathrm{~ms}$ ) at $5 \mathrm{~Hz}$ given in a continuous train and an intensity of $80 \%$ aMT ( $40 \mathrm{~s}$ in total). Finally, both groups performed another set of motor-training interventions (mirror task), and the motor functions were measured again (Post3). One set of behavioral and TMS measurements took $\sim 5 \mathrm{~min}$, so the whole experiment was completed within 15-20 min after cTBS.

Data analysis. For Experiment 1, a two-way repeated-measures ANOVA was used with Intervention (Pre and Post) as a within-subject factor and Group (mirror task and nonmirror task) as a between-subject factor. For I-O function, Intensity (50, 80, 90,100, 110, 120, 130, and $150 \%$ ) was used as a within-subject factor. For Experiment 2, a two-way repeated-measures ANOVA was used for each hand with Intervention (Pre and Post) as a within-subject factor and Group (mirror task, nonmirror task, and AO task) as a between-subject factor. For Experiment 3, a two-way repeated-measures ANOVA was used with Time (Pre, Post1, Post2, and Post3) as a within-subject factor and Site (M1 and OC) as a between-subject factor. If necessary, the Greenhouse-Geisser correction was used to adjust for sphericity, changing the degrees of freedom using a correction coefficient $\varepsilon$. The Bonferroni correction for multiple comparisons was used for the post hoc $t$ test. Effects were considered significant at $p<0.05$. All data are given as the mean \pm SEM. 


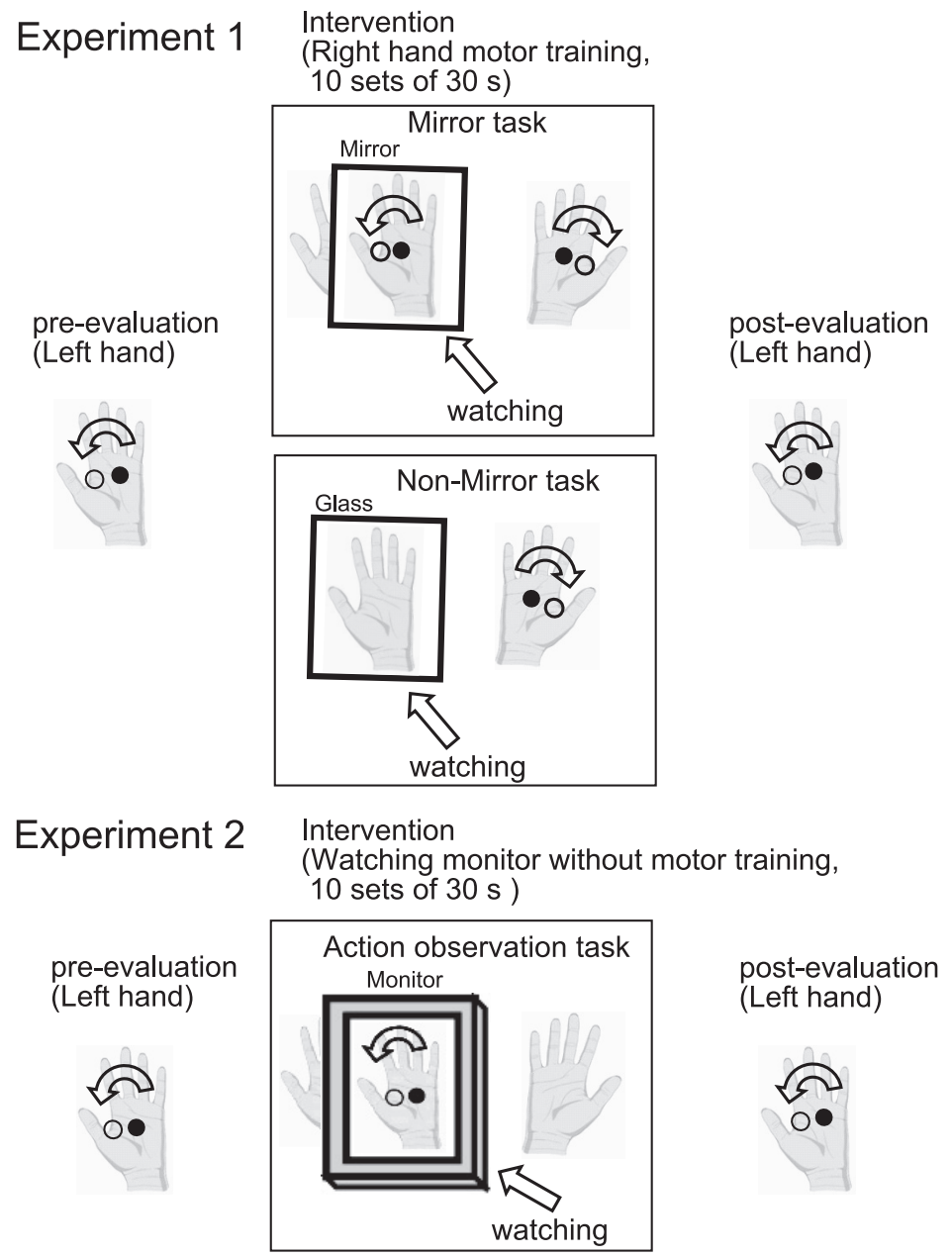

Experiment 3

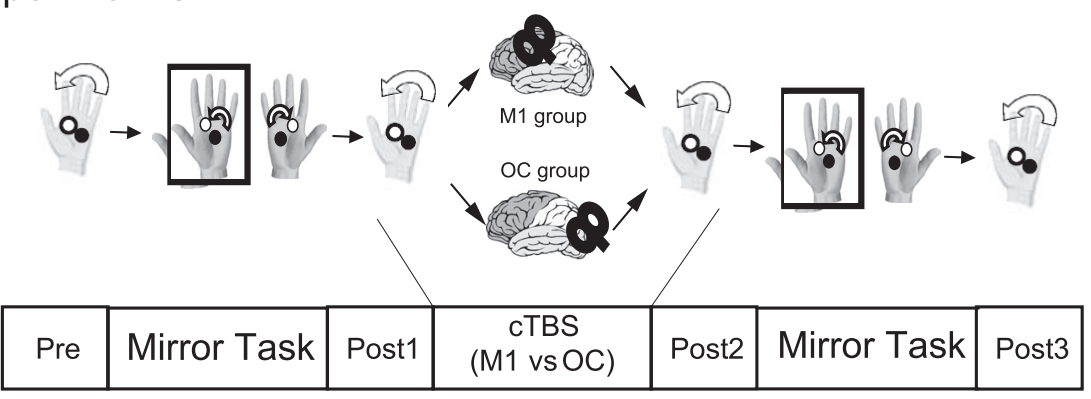

Figure 1. Task design. In Experiment 1, behavioral and electrophysiological measurements were examined before and after each motor-training intervention (mirror and nonmirror tasks). In Experiment 2, behavioral measurements in both hands were examined before and after the $\mathrm{A} 0$ task as well as mirror and nonmirror tasks. In Experiment 3, functional interference using cTBS was applied to the right $\mathrm{M} 1$ or OC just after the mirror task. Both groups then performed another set of mirror tasks. The motor function of the left hand was measured before and after the first mirror task, after cTBS, and after the second mirror task (Pre, Post1, Post2, and Post3, respectively).

\section{Results}

\section{Experiment 1: mirror versus nonmirror tasks}

Behavioral measurements

Two-way repeated-measures ANOVA showed significant effects of Intervention $(F=39.6, p<0.001)$ and Intervention $\times$ Group interactions $(F=12.9, p=0.002)$ for the number of ball rotations. Post hoc $t$ test analysis revealed a significant behavioral improvement after motor-training intervention in the mirror group $(p<0.001)$ but not in the nonmirror group (Fig. 2A).

\section{TMS measurements}

The mean rMTs for the left FDI in the mirror and nonmirror groups preintervention were $54.0 \pm 3.1 \%$ and $56.6 \pm 3.9 \%$, respectively, of the maximum stimulator output; postintervention, they were $53.3 \pm 3.3 \%$ and $56.8 \pm$ $3.6 \%$, respectively. Two-way repeatedmeasures ANOVA showed no significant effects of Intervention or Intervention $\times$ Group interactions.

The mean aMTs for the left FDI in the mirror and nonmirror groups were $43.3 \pm 2.8 \%$ and $43.8 \pm 2.6 \%$, respectively, preintervention, and $40.7 \pm 3.0 \%$ and $42.9 \pm 2.3 \%$, respectively, postintervention. In this case, two-way repeatedmeasures ANOVA showed a significant effect of Intervention $(F=28.1, p<$ $0.001)$ and Intervention $\times$ Group interactions $(F=6.62, p=0.019)$. The post hoc $t$ test revealed a significant decrease after motor-training intervention in the mirror group $(p<0.001)$ but not in the nonmirror group.

The mean MEP amplitudes for the left FDI in the mirror and nonmirror groups were $1250 \pm 167 \mu \mathrm{V}$ and $987 \pm 159 \mu \mathrm{V}$, respectively, preintervention, and $1785 \pm 236$ $\mu \mathrm{V}$ and $967 \pm 168 \mu \mathrm{V}$, respectively, postintervention. Two-way repeated-measures ANOVA showed significant effects of Intervention $(F=13.8, p=0.002)$ and Intervention $\times$ Group interactions $(F=16.1$, $p=0.001)$, whereas the post hoc $t$ test revealed a significant increase after motortraining intervention in the mirror group $(p=0.002)$ but not in the nonmirror group (Fig. $2 B, C$ ).

For the I-O function of MEP amplitudes, the results from one subject in the mirror group and two subjects in the nonmirror group were discarded from the analysis because a technical error occurred during recording. Therefore, I-O function data were analyzed from nine subjects in the mirror group and eight subjects in the nonmirror group. For the mirror group, two-way repeated-measures ANOVA showed significant effects of Intervention $(F=14.7, p=0.005)$, Intensity $(F=52.6, p<0.001)$, and Intervention $\times$ Intensity interactions $(F=5.5$, $p=0.003)$, suggesting that MEP amplitudes as a function of TMS intensity were significantly different postintervention compared with preintervention (Fig. 3). Post hoc $t$ testing revealed a significant change in MEP amplitude after intervention at an intensity of $120 \%(p=0.019)$. However, the original paired $t$ test for the I-O function showed a significant difference at $130 \%$ and $150 \%(p=0.048$ and 0.018 , respectively, without correcting for multiple comparisons). For the nonmirror group, two-way repeated-measures ANOVA showed no significant effects of Intervention, Intensity, or Intervention $\times$ Intensity interactions.

The SICI was $0.464 \pm 0.044$ in the mirror group and $0.512 \pm$ 0.070 in the nonmirror group preintervention, and $0.590 \pm 0.067$ and $0.529 \pm 0.115$, respectively, postintervention. Two-way 
A

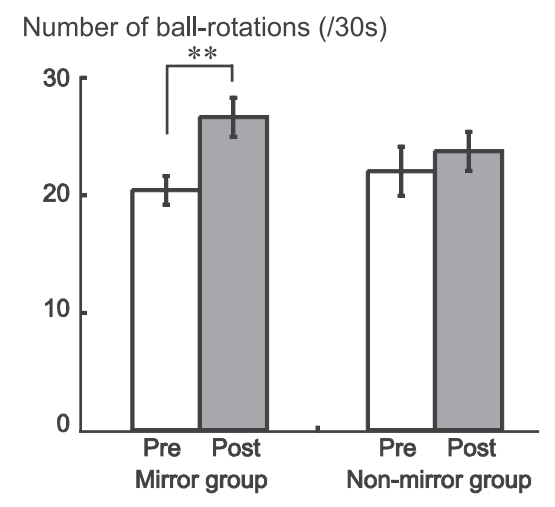

B
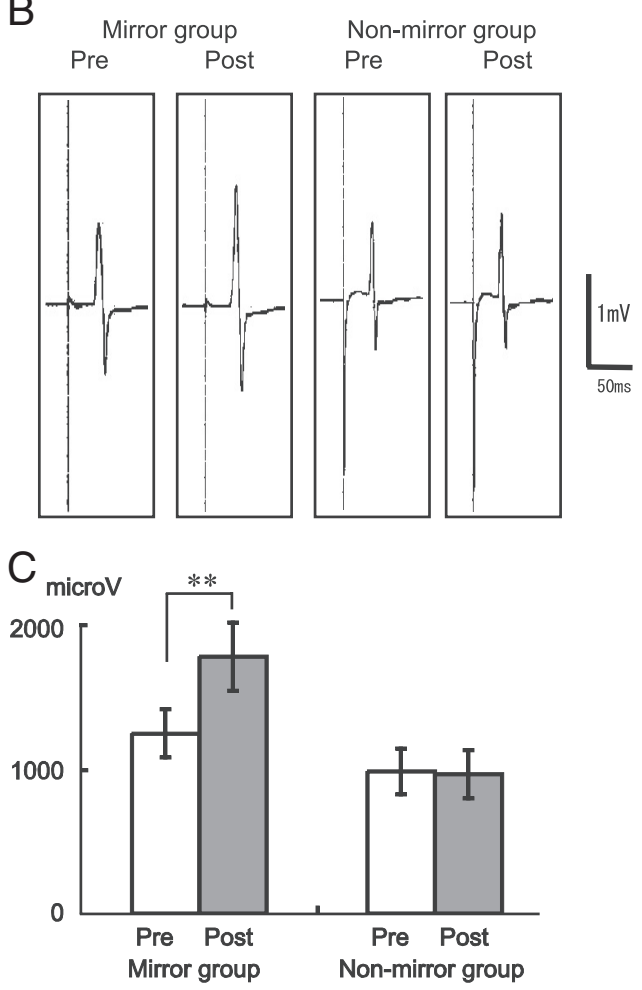

Figure 2. Effects of mirror and nonmirror tasks on right $M 1$ function. $A$, The mean number of ball rotations performed by the left hand increased significantly after the mirror task. $\boldsymbol{B}$, The average MEP of the left FDI (single subject) increased after the mirror task. $\boldsymbol{C}$, The mean MEP amplitude was significantly enlarged after the mirror task. ${ }^{* *} p<0.001$.

repeated-measures ANOVA showed no significant effects of Intervention or Intervention $\times$ Group interactions.

The IHI(10) from the left to the right M1 was recorded preintervention as $0.616 \pm 0.070$ in the mirror group and $0.782 \pm 0.038$ in the nonmirror group, and as $0.628 \pm 0.066$ and $0.701 \pm 0.076$, respectively, postintervention. Preintervention, the $\mathrm{IHI}(40)$ was $0.824 \pm 0.035$ in the mirror group and $0.902 \pm 0.045$ in the nonmirror group, and $0.905 \pm 0.091$ and $0.836 \pm 0.058$, respectively, postintervention. Two-way repeated-measures ANOVA showed no significant effects of Intervention and Intervention $\times$ Intensity interactions in either $\mathrm{IHI}(10)$ or $\mathrm{IHI}(40)$.

F-wave

The mean F-wave amplitudes in the left FDI before and after intervention in the mirror group were not significantly different at $205 \pm 56 \mu \mathrm{V}$ and $222 \pm 56 \mu \mathrm{V}$, respectively.

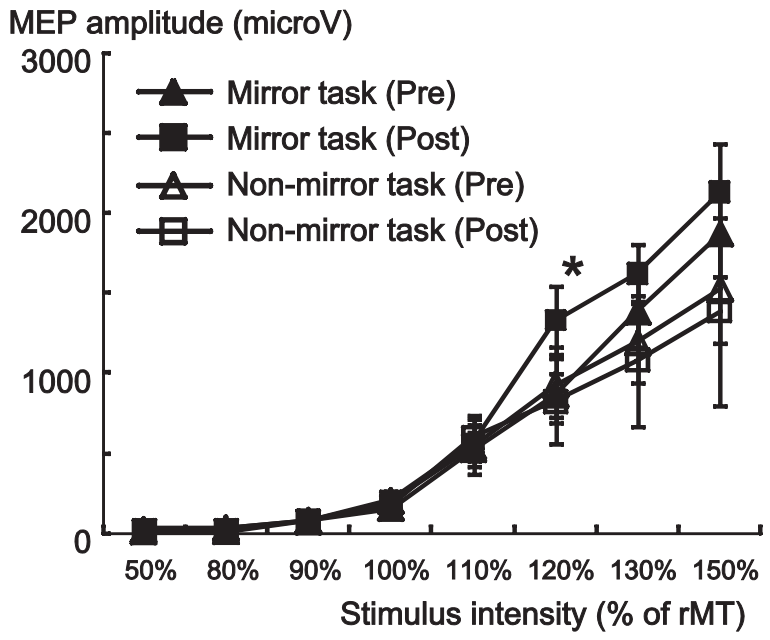

Figure 3. MEP amplitudes as a function of TMS intensity preintervention and postintervention. The MEP amplitude of the left FDI significantly increased after the mirror task at $120 \%$ of the resting MT in the mirror group. ${ }^{*} p<0.05$.
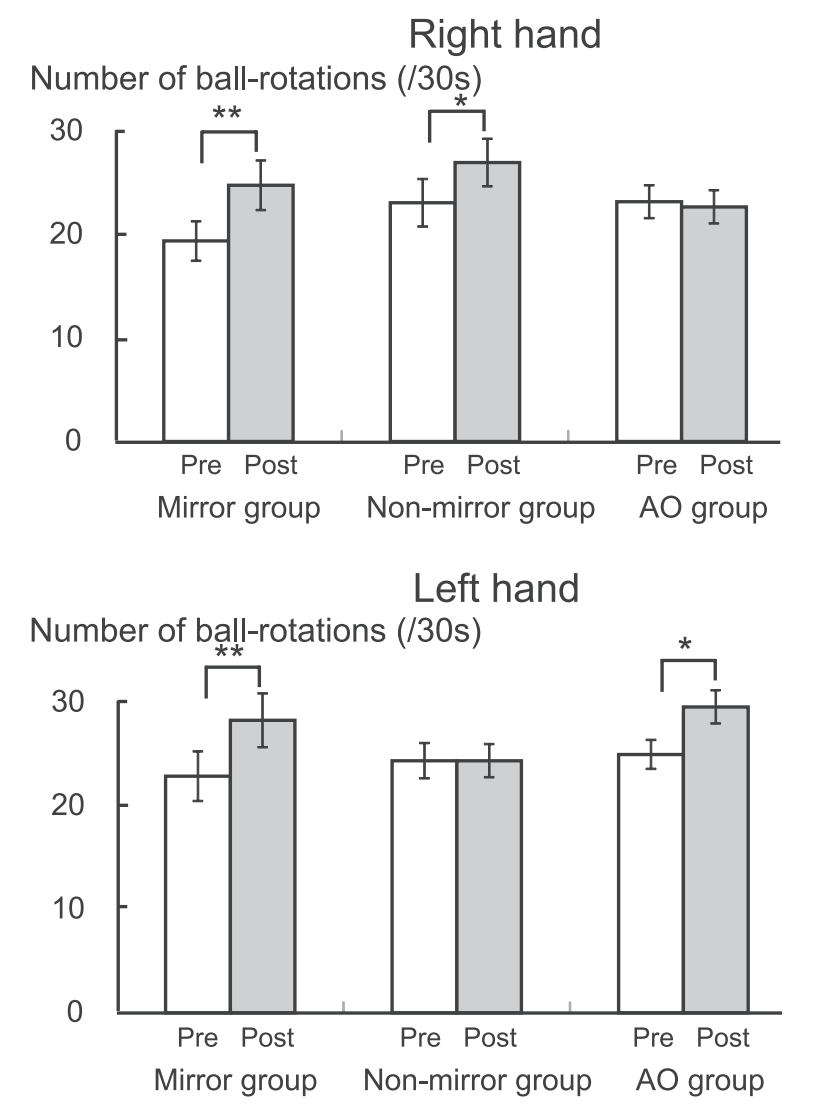

Figure 4. Effects of $\mathrm{A} 0$, mirror, and nonmirror tasks on right and left $\mathrm{M} 1 \mathrm{functions}$. The mean number of ball rotations increased significantly after the mirror and A0 tasks in the left hand, and after the mirror and nonmirror tasks in the right hand. ${ }^{*} p<0.05,{ }^{* *} p<0.001$.

\section{Experiment 2: mirror versus $\mathrm{AO}$ tasks}

For the left hand, two-way repeated-measures ANOVA showed significant effects of Intervention $(F=49.2, p<0.001)$ and Intervention $\times$ Group interactions $(F=12.6, p<0.001)$ for the number of ball rotations. Post hoc $t$ testing revealed a significant behavioral improvement after motor-training intervention in the mirror group $(p=0.001)$ and the $\mathrm{AO}$ group $(p=0.023)$, but not in the nonmirror group (Fig. 4). 


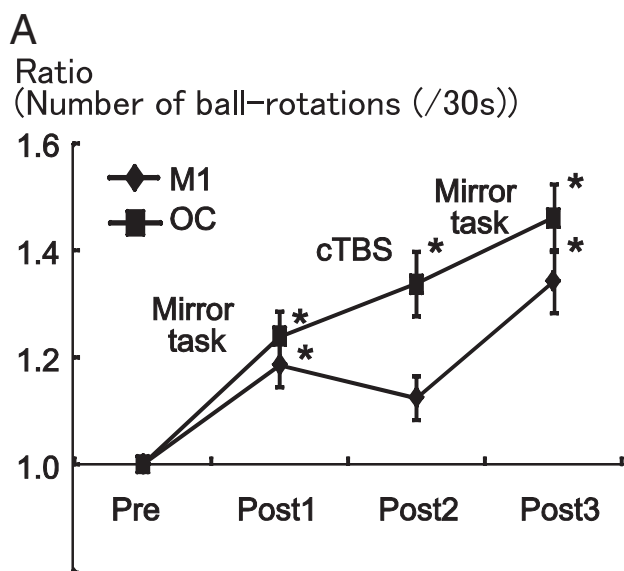

B

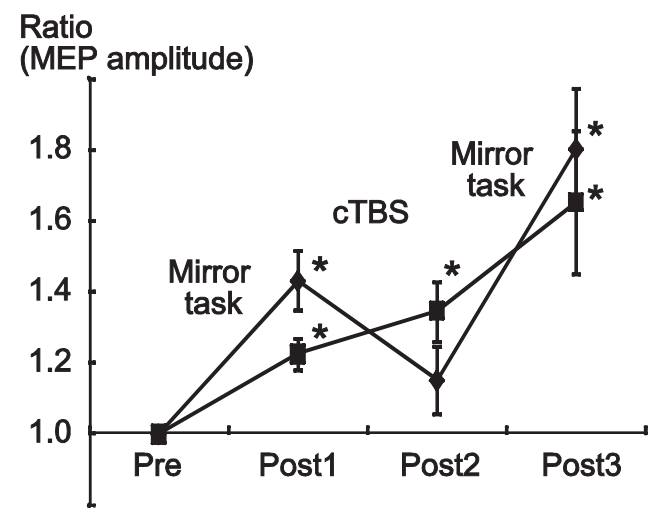

Figure 5. Effects of CTBS on MVF-induced changes. $A$, For the CTBS at the M1, the mean number of ball rotations performed by the left hand increased significantly after the first and second mirror tasks (Post1 and Post3), but not after CTBS (Post2). For the cTBS at OC, it increased monotonously (Post1, Post2, and Post3 > Pre). B, The mean MEP amplitude showed a similar pattern of behavior, with significant increases at Post 1 and Post 3 compared with Pre for the M1 group, and at Post1, Post2, and Post3 for the 0 C group. . $p<0.001$.

For the right hand, two-way repeated-measures ANOVA showed significant effects of Intervention $(F=23.0, p<0.001)$ and Intervention $\times$ Group interactions $(F=8.6, p<0.001)$. Post hoc $t$ testing revealed a significant behavioral improvement after motor-training intervention in the mirror group $(p=0.001)$ and nonmirror group ( $p=0.029)$, but not the AO group.

\section{Experiment 3: effects of cTBS}

\section{Behavioral measurements}

Two-way repeated-measures ANOVA showed significant effects of Time $(F=81.3, p<0.001)$ and Time $\times$ Site $(F=8.3, p=$ 0.002 ) interactions for the number of ball rotations. Post hoc $t$ testing revealed a significant behavioral improvement in Post1 $(p=0.002)$ and Post3 $(p<0.001)$, but not in Post2, compared with Pre in the M1 group. A significant behavioral improvement was also found in Post1 $(p=0.002)$, Post2 $(p<0.001)$, and Post3 $(p<0.001)$ compared with Pre in the OC group (Fig. 5A).

\section{TMS measurements}

The mean rMTs for the left FDI were not significantly different in the M1 and OC groups before the intervention at $43.8 \pm 8.0 \%$ and $53.1 \pm 10.4 \%$, respectively, of the maximum stimulator output.

The mean MEP amplitudes were $801 \pm 178,869 \pm 195,801 \pm$ 178 , and $1362 \pm 259 \mu \mathrm{V}$ for the M1 group, and $780 \pm 178,929 \pm$ $170,969 \pm 153$, and $1114 \pm 176 \mu \mathrm{V}$ for the OC group. Two-way
MEP ratio

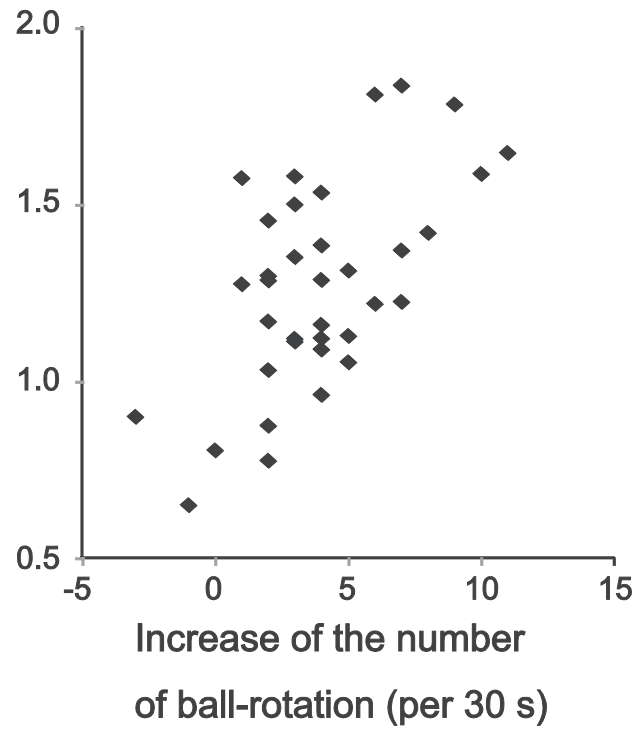

Figure 6. Correlation between M1 plasticity and behavioral improvements. The MEP ratio and the change in number of ball rotations were significantly correlated (Pearson's correlation, $r=0.603, p<0.001)$.

repeated-measures ANOVA for MEP amplitudes revealed significant effects of Time $(F=22.0, p<0.001)$ and Time $\times$ Site interactions $(F=3.4, p=0.026)$. Post hoc $t$ testing revealed a significant increase after the motor-training intervention in Post1 $(p=0.007)$ and Post3 $(p=0.002)$, but not in Post2, compared with Pre in the M1 group. A significant increase was also found in Post1 $(p=0.03)$, Post2 $(p=0.005)$, and Post3 ( $p=$ 0.01 ) compared with Pre in the OC group (Fig. $5 B$ ).

Correlation between M1 plasticity and behavioral improvement To investigate the correlation between the increase in MEP amplitudes and improved motor behavior, we calculated the Pearson's correlation coefficient for all subjects between the MEP ratio and the change in number of ball rotations, and found it to be significant $(r=0.603, p<0.001)$. For the MEP ratio, the MEP amplitude after motor-training intervention was divided by the MEP amplitude preintervention. The change in motor behaviors was similarly measured before and after motor-training intervention (Fig. 6).

\section{Discussion}

The present study showed that repetitive motor training of the right hand significantly improved the motor performance of the left hand if MVF was also given. This behavioral improvement was associated with facilitation of the excitatory function of the corticospinal pathway, which increased the MEP amplitude and its I-O function, and decreased the aMT. However, there was no significant change in the inhibitory function of the right M1, such as the SICI or IHI. Spinal motoneuronal excitability, as measured by the F-wave, was insensitive to MVF, suggesting a supraspinal mechanism for this plasticity. Thus, it is likely that the behavioral improvement induced by MVF was caused by a plastic change in the right M1 via its excitatory connections.

Correlation analysis revealed a significant positive relationship between behavioral improvement and the MEP increase across all subjects, whereas the right M1 interference using cTBS disrupted the effect of MVF both behaviorally and electrophysiologically. These findings further support the above-mentioned 
hypothesis, and reveal a close link between the right M1 plasticity and behavioral improvement of the left hand in MVF.

The motor performance of both hands improved with MVF to the same extent. However, only the performance of the right hand improved for the nonmirror task, and only that of the left hand improved when a recording of the left hand was viewed without any actual motor training. Thus, it is likely that visual feedback provided by the mirror is relevant for the motor improvement of the stationary hand, rather than intermanual transfer from right hand. Moreover, since there was no motor improvement in the right hand when visual feedback was given over the left hand during the action observation task, the effects of MVF-induced motor improvement may be effector-specific. It is highly possible that the beneficial effects of mirror therapy might be associated with visual feedback similar to action observation.

Previous physiological studies using TMS (Garry et al., 2005) or fMRI (Shinoura et al., 2008) investigated brain function during motor training with MVF and showed M1 activation ipsilateral to the moving hand. Changes in M1 function of the stationary hand after motor training with MVF have so far not been studied, yet they are of great relevance for the purpose of rehabilitation. A recent fMRI study in stroke patients (Michielsen et al., 2011) showed that the mirror illusion during a bimanual, but not unimanual, task was associated with increased activity in the precuneus and the posterior cingulate cortex (Movement $\times$ Mirror interaction), and that the main effect of Mirror showed no significant areas of activation. Thus, it is possible that those areas might be associated with MVF-induced M1 plasticity. This study differed from our own in that it investigated brain activation during unimanual and bimanual motor training with MVF, whereas we focused on the change of M1 function of the stationary hand before and after motor training with MVF.

By applying a relatively complex motor-skill learning task, we showed that behavioral and electrophysiological improvements were induced in normal subjects only when MVF was combined with motor training.

To clarify the role of visual feedback and intermanual transfer, Experiment 2 showed that the improvement in the left hand could be induced by visual feedback alone without the right-hand movement (AO task). In contrast, the improvement of the right hand was associated with actual motor training (mirror and nonmirror tasks). Thus, the motor improvement caused by MVF might not be due to intermanual learning transfer from the right to the left hand.

Although intermanual transfer was observed previously (Halsband, 1992; Criscimagna-Hemminger et al., 2003; van Mier and Petersen, 2006; Wang et al., 2011), it was not found in the nonmirror task of the present study. This might have been because asymmetrical intermanual transfer occurred instead when using the symmetric mirror motor task (Kirsch and Hoffmann, 2010). However, it should be noted that the pattern of intermanual transfer might differ between healthy controls and stroke patients. Indeed, a recent study reported the consistent healthyto-affected transfer of motor skill regardless of hand dexterity (Ausenda and Carnovali, 2011).

In addition to providing visual feedback, it is possible that the MVF created intermodal conflict between visual and proprioceptive and tactile senses. The right dorsolateral prefrontal cortex (DLPFC) was previously shown to be activated in the face of intermodal conflicting visual feedback from a mirror when subjects looked at the reflection of their right hand (Fink et al., 1999).
As the mirror and $\mathrm{AO}$ tasks were associated with intermodal conflict, both this and MVF might have affected M1 plasticity via DLPFC activation.

Regarding increased right M1 excitability, the present study showed that the active MTs were significantly decreased, and MEP amplitudes as a function of stimulus intensity (I-O function) were significantly changed in the mirror group. The MT and I-O function might provide information on different aspects of motor cortical excitability (Hallett et al., 1999). The MT reflects membrane-related intrinsic neuronal excitability (Ziemann et al., 1996a), whereas the I-O function is related to more global measures of excitability of the corticospinal pathways (Devanne et al., 1997).

Although the aMT, reflecting the neuronal membrane excitability levels in the M1 (Mavroudakis et al., 1994; Ziemann et al., 1996a; Hallett, 2000), was significantly decreased after mirror therapy, the change in the rMT failed to reach statistical significance. Indeed, the mean rMT was reduced slightly after MVF (from $54 \%$ to $53 \%$ ). This apparent discrepancy could be due to intersubject rMT variability, as the rMT is determined by several processes, including membrane excitability, corticocortical axon excitability, synaptic efficacy between axons and corticospinal neurons, and spinal connectivity.

The I-O function reflects not only the number of firing neurons activated by the suprathreshold stimuli, but also the neuronal excitability produced by the subthreshold stimuli (Ridding and Rothwell, 1997), suggesting a cortical mechanism.

Although neuronal plasticity has been recognized to occur at multiple levels of the CNS, the change induced by MVF in the present study was likely to be at the supraspinal level, as there was no significant change to the F-wave. These results suggest that changes of corticospinal excitability induced by MVF might originate in the right M1. By contrast, there was no significant change in the inhibitory function of the right M1. Although the SICI system, which partly reflects $\mathrm{GABA}_{\mathrm{A}}$-mediated inhibition, plays an important role in changing M1 excitability and motor performance (Chen, 2004; Ziemann, 2004), the MVF did not change the SICI significantly.

Several previous studies showed that IHI modulation might contribute to the intermanual transfer of a force-control task (Perez et al., 2007; Camus et al., 2009), but the change of connectivity between bilateral M1 regions is not likely to be relevant for MVF.

The TBS, which modulates M1 excitability in an inhibitory or excitatory way depending on the stimulation pattern (Huang and Rothwell, 2004; Huang et al., 2005; Gamboa et al., 2010), consists of three pulses at $50 \mathrm{~Hz}$ repeated at $5 \mathrm{~Hz}$. Intermittent TBS increases M1 excitability, whereas cTBS decreases it (Huang et al., 2005); cTBS after-effects include MEP suppression for $>20 \mathrm{~min}$ (Gentner et al., 2008). In the present study, the motor learning effect was disrupted immediately by the interference over the M1 but not the OC. Reduced motor dexterity could be relearned after the second mirror task. It was reported that $1 \mathrm{~Hz}$ rTMS over the M1 could disrupt behavioral improvement (Muellbacher et al., 2002). In addition, a recent report suggested that $1 \mathrm{~Hz}$ rTMS could disturb motor learning by observation (Brown et al., 2009; Censor and Cohen, 2011). It is possible that the interference effect produced by cTBS was similar to that produced by $1 \mathrm{~Hz}$ rTMS, as both protocols suppressed M1 activity.

As cTBS over the M1 can suppress basal motor function (Huang and Rothwell, 2004; Huang et al., 2005; Gamboa et al., 2010), we cannot rule out the possibility that the cTBS-induced disruption of the right M1 plasticity in the present study might 
have been caused by suppression of the M1 function in general but not by the disruption of motor learning. The effects of the mirror task were disturbed after cTBS and restored after relearning, suggesting a commonly shared neural mechanism for cTBS-induced M1 plasticity and MVF-related motor plasticity. However, we cannot exclude the possibility that other motor-related areas, such as supplementary motor areas, dorsal premotor areas, or DLPFC, might play an important role in MVF-related M1 plasticity.

Recent studies applying cTBS over the M1 before motor practice showed that it could disturb the early motor learning of simple finger movements (Iezzi et al., 2010) and implicit sequence learning (Wilkinson et al., 2010). However, in the present study, the motor learning effect produced by the second motor practice after cTBS remained unchanged, whereas the early motor retention produced by the first motor practice before cTBS was disrupted. This divergence might be due to differences in the used motor tasks.

If we had evaluated paired-pulse intracortical facilitation $(\mathrm{Ku}-$ jirai et al., 1993) and paired-pulse short-interval intracortical facilitation (Tokimura et al., 1996; Ziemann et al., 1998), the results might have provided valuable information about the excitatory connections of the right $\mathrm{M} 1$; however, this could not be performed because of time limitations on our experiment that were established to avoid subject fatigue.

Several hypotheses have been proposed to explain the mechanism by which right M1 plasticity is produced by MVF. One is the implicit motor imagery during MVF. As M1 activation has been reported during motor imagery (Kasai et al., 1997; Hashimoto and Rothwell, 1999; Hanakawa et al., 2008), it is possible that MVF might further facilitate this imagery mechanism.

Another possible mechanism involves the mirror neuron system (MNS) (Rosén and Lundborg, 2005; Sütbeyaz et al., 2007). The primate MNS is a frontoparietal motor network of bimodal visuomotor mirror neurons that discharge when they perform a particular action and when observing a similar action performed by another (Rizzolatti and Craighero, 2004). The human M1 has MNS-like properties, because its facilitation during action observation is effector-specific (Maeda et al., 2002), lateralized (AzizZadeh et al., 2002), and significantly greater in a first-person perspective than a third-person perspective (Fadiga et al., 2005; Sartori et al., 2011). In one fMRI study (Matthys et al., 2009), the superior temporal gyrus was activated during MVF intervention, suggesting a link between MVF and MNS. Therefore, it is also possible that the increased right M1 excitability induced by MVF might be caused by its MNS-like properties. However, regardless of either mechanism, our study clearly showed that the final common pathway for the effect of MVF is the plastic change in the M1. The effects of MVF on corticospinal excitability and motor behavior, and especially on hand dexterity, suggest that it might be useful in stroke rehabilitation.

\section{References}

Altschuler EL, Wisdom SB, Stone L, Foster C, Galasko D, Llewellyn DM, Ramachandran VS (1999) Rehabilitation of hemiparesis after stroke with a mirror. Lancet 353:2035-2036.

Ausenda C, Carnovali M (2011) Transfer of motor skill learning from the healthy hand to the paretic hand in stroke patients: a randomized controlled trial. Eur J Phys Rehabil Med 47:417-425.

Aziz-Zadeh L, Maeda F, Zaidel E, Mazziotta J, Iacoboni M (2002) Lateralization in motor facilitation during action observation: a TMS study. Exp Brain Res 144:127-131.

Brown LE, Wilson ET, Gribble PL (2009) Repetitive transcranial magnetic stimulation to the primary motor cortex interferes with motor learning by observing. J Cogn Neurosci 21:1013-1022.

Camus M, Ragert P, Vandermeeren Y, Cohen LG (2009) Mechanisms con- trolling motor output to a transfer hand after learning a sequential pinch force skill with the opposite hand. Clin Neurophysiol 120:1859-1865.

Censor N, Cohen LG (2011) Using repetitive transcranial magnetic stimulation to study the underlying neural mechanisms of human motor learning and memory. J Physiol 589:21-28.

Chen R (2004) Interactions between inhibitory and excitatory circuits in the human motor cortex. Exp Brain Res 154:1-10.

Chen R, Yung D, Li JY (2003) Organization of ipsilateral excitatory and inhibitory pathways in the human motor cortex. J Neurophysiol 89:1256-1264.

Criscimagna-Hemminger SE, Donchin O, Gazzaniga MS, Shadmehr R (2003) Learned dynamics of reaching movements generalize from dominant to nondominant arm. J Neurophysiol 89:168-176.

Devanne H, Lavoie BA, Capaday C (1997) Input-output properties and gain changes in the human corticospinal pathway. Exp Brain Res 114:329-338.

Fadiga L, Craighero L, Olivier E (2005) Human motor cortex excitability during the perception of others' action. Curr Opin Neurobiol 15:213-218.

Ferbert A, Priori A, Rothwell JC, Day BL, Colebatch JG, Marsden CD (1992) Interhemispheric inhibition of the human motor cortex. J Physiol 453:525-546.

Fink GR, Marshall JC, Halligan PW, Frith CD, Driver J, Frackowiak RS, Dolan RJ (1999) The neural consequences of conflict between intention and the senses. Brain 122:497-512.

Gamboa OL, Antal A, Moliadze V, Paulus W (2010) Simply longer is not better: reversal of theta burst after-effect with prolonged stimulation. Exp Brain Res 204:181-187.

Garry MI, Loftus A, Summers JJ (2005) Mirror, mirror on the wall: viewing a mirror reflection of unilateral hand movements facilitates ipsilateral M1 excitability. Exp Brain Res 163:118-122.

Gentner R, Wankerl K, Reinsberger C, Zeller D, Classen J (2008) Depression of human corticospinal excitability induced by magnetic theta-burst stimulation: evidence of rapid polarity-reversing metaplasticity. Cereb Cortex 18:2046-2053.

Giraux P, Sirigu A (2003) Illusory movements of the paralyzed limb restore motor cortex activity. Neuroimage 20 [Suppl 1]:S107-S111.

Hallett M (2000) Transcranial magnetic stimulation and the human brain. Nature 406:147-150.

Hallett M, Chen R, Ziemann U, Cohen LG (1999) Reorganization in motor cortex in amputees and in normal volunteers after ischemic limb deafferentation. Electroencephalogr Clin Neurophysiol Suppl 51:183-187.

Halsband U (1992) Left hemisphere preponderance in trajectorial learning. Neuroreport 3:397-400.

Hanakawa T, Dimyan MA, Hallett M (2008) Motor planning, imagery, and execution in the distributed motor network: a time-course study with functional MRI. Cereb Cortex 18:2775-2788.

Hashimoto R, Rothwell JC (1999) Dynamic changes in corticospinal excitability during motor imagery. Exp Brain Res 125:75-81.

Huang YZ, Rothwell JC (2004) The effect of short-duration bursts of highfrequency, low-intensity transcranial magnetic stimulation on the human motor cortex. Clin Neurophysiol 115:1069-1075.

Huang YZ, Edwards MJ, Rounis E, Bhatia KP, Rothwell JC (2005) Theta burst stimulation of the human motor cortex. Neuron 45:201-206.

Iezzi E, Suppa A, Conte A, Agostino R, Nardella A, Berardelli A (2010) Theta-burst stimulation over primary motor cortex degrades early motor learning. Eur J Neurosci 31:585-592.

Kasai T, Kawai S, Kawanishi M, Yahagi S (1997) Evidence for facilitation of motor evoked potentials (MEPs) induced by motor imagery. Brain Res 744:147-150.

Kirsch W, Hoffmann J (2010) Asymmetrical intermanual transfer of learning in a sensorimotor task. Exp Brain Res 202:927-934.

Koganemaru S, Mima T, Nakatsuka M, Ueki Y, Fukuyama H, Domen K (2009) Human motor associative plasticity induced by paired bihemispheric stimulation. J Physiol 587:4629-4644.

Koganemaru S, Mima T, Thabit MN, Ikkaku T, Shimada K, Kanematsu M, Takahashi K, Fawi G, Takahashi R, Fukuyama H, Domen K (2010) Recovery of upper-limb function due to enhanced use-dependent plasticity in chronic stroke patients. Brain 133:3373-3384.

Kujirai T, Caramia MD, Rothwell JC, Day BL, Thompson PD, Ferbert A, Wroe S, Asselman P, Marsden CD (1993) Corticocortical inhibition in human motor cortex. J Physiol 471:501-519.

Maeda F, Kleiner-Fisman G, Pascual-Leone A (2002) Motor facilitation 
while observing hand actions: specificity of the effect and role of observer's orientation. J Neurophysiol 87:1329-1335.

Matthys K, Smits M, Van der Geest JN, Van der Lugt A, Seurinck R, Stam HJ, Selles RW (2009) Mirror-induced visual illusion of hand movements: a functional magnetic resonance imaging study. Arch Phys Med Rehabil 90:675-681.

Mavroudakis N, Caroyer JM, Brunko E, Zegers de Beyl D (1994) Effects of diphenylhydantoin on motor potentials evoked with magnetic stimulation. Electroencephalogr Clin Neurophysiol 93:428-433.

McCabe CS, Haigh RC, Ring EF, Halligan PW, Wall PD, Blake DR (2003) A controlled pilot study of the utility of mirror visual feedback in the treatment of complex regional pain syndrome (type 1). Rheumatology (Oxford) 42:97-101.

Mercuri B, Wassermann EM, Manganotti P, Ikoma K, Samii A, Hallett M (1996) Cortical modulation of spinal excitability: an F-wave study. Electroencephalogr Clin Neurophysiol 101:16-24.

Michielsen ME, Smits M, Ribbers GM, Stam HJ, van der Geest JN, Bussmann JB, Selles RW (2011) The neuronal correlates of mirror therapy: an fMRI study on mirror induced visual illusions in patients with stroke. J Neurol Neurosurg Psychiatry 82:393-398.

Moseley GL (2004) Graded motor imagery is effective for long-standing complex regional pain syndrome: a randomised controlled trial. Pain 108:192-198.

Muellbacher W, Ziemann U, Wissel J, Dang N, Kofler M, Facchini S, Boroojerdi B, Poewe W, Hallett M (2002) Early consolidation in human primary motor cortex. Nature 415:640-644.

Oishi N, Mima T, Ishii K, Bushara KO, Hiraoka T, Ueki Y, Fukuyama H, Hallett M (2007) Neural correlates of regional EEG power change. Neuroimage 36:1301-1312.

Oldfield RC (1971) The assessment and analysis of handedness: the Edinburgh inventory. Neuropsychologia 9:97-113.

Pascual-Leone A, Tormos JM, Keenan J, Tarazona F, Cañete C, Catalá MD (1998) Study and modulation of human cortical excitability with transcranial magnetic stimulation. J Clin Neurophysiol 15:333-343.

Perez MA, Wise SP, Willingham DT, Cohen LG (2007) Neurophysiological mechanisms involved in transfer of procedural knowledge. J Neurosci 27:1045-1053.

Ramachandran VS, Rogers-Ramachandran D (1996) Synaesthesia in phantom limbs induced with mirrors. Proc Biol Sci 263:377-386.

Ridding MC, Rothwell JC (1997) Stimulus/response curves as a method of measuring motor cortical excitability in man. Electroencephalogr Clin Neurophysiol 105:340-344.

Rizzolatti G, Craighero L (2004) The mirror-neuron system. Annu Rev Neurosci 27:169-192.

Rosén B, Lundborg G (2005) Training with a mirror in rehabilitation of the hand. Scand J Plast Reconstr Surg Hand Surg 39:104-108.

Rossini PM, Barker AT, Berardelli A, Caramia MD, Caruso G, Cracco RQ, Dimitrijević MR, Hallett M, Katayama Y, Lücking CH (1994) Noninvasive electrical and magnetic stimulation of the brain, spinal cord and roots: basic principles and procedures for routine clinical application: report of an IFCN committee. Electroencephalogr Clin Neurophysiol 91:79-92.

Rothwell JC, Hallett M, Berardelli A, Eisen A, Rossini P, Paulus W (1999) Magnetic stimulation: motor evoked potentials. The International Feder- ation of Clinical Neurophysiology. Electroencephalogr Clin Neurophysiol Suppl 52:97-103.

Sartori L, Cavallo A, Bucchioni G, Castiello U (2011) Corticospinal excitability is specifically modulated by the social dimension of observed actions. Exp Brain Res 211:557-568.

Sathian K, Greenspan AI, Wolf SL (2000) Doing it with mirrors: a case study of a novel approach to neurorehabilitation. Neurorehabil Neural Repair $14: 73-76$

Shinoura N, Suzuki Y, Watanabe Y, Yamada R, Tabei Y, Saito K, Yagi K (2008) Mirror therapy activates outside of cerebellum and ipsilateral M1. NeuroRehabilitation 23:245-252.

Stevens JA, Stoykov ME (2003) Using motor imagery in the rehabilitation of hemiparesis. Arch Phys Med Rehabil 84:1090-1092.

Sütbeyaz S, Yavuzer G, Sezer N, Koseoglu BF (2007) Mirror therapy enhances lower-extremity motor recovery and motor functioning after stroke: a randomized controlled trial. Arch Phys Med Rehabil 88:555-559.

Thabit MN, Ueki Y, Koganemaru S, Fawi G, Fukuyama H, Mima T (2010) Movement-related cortical stimulation can induce human motor plasticity. J Neurosci 30:11529-11536.

Tokimura H, Ridding MC, Tokimura Y, Amassian VE, Rothwell JC (1996) Short latency facilitation between pairs of threshold magnetic stimuli applied to human motor cortex. Electroencephalogr Clin Neurophysiol 101:263-272.

Tominaga W, Matsubayashi J, Deguchi Y, Minami C, Kinai T, Nakamura M, Nagamine T, Matsuhashi M, Mima T, Fukuyama H, Mitani A (2009) A mirror reflection of a hand modulates stimulus-induced $20-\mathrm{Hz}$ activity. Neuroimage 46:500-504.

van Mier HI, Petersen SE (2006) Intermanual transfer effects in sequential tactuomotor learning: evidence for effector independent coding. Neuropsychologia 44:939-949.

Wang J, Joshi M, Lei Y (2011) The extent of interlimb transfer following adaptation to a novel visuomotor condition does not depend on awareness of the condition. J Neurophysiol 106:259-264.

Wilkinson L, Teo JT, Obeso I, Rothwell JC, Jahanshahi M (2010) The contribution of primary motor cortex is essential for probabilistic implicit sequence learning: evidence from theta burst magnetic stimulation. J Cogn Neurosci 22:427-436.

Yavuzer G, Selles R, Sezer N, Sütbeyaz S, Bussmann JB, Köseoğlu F, Atay MB, Stam HJ (2008) Mirror therapy improves hand function in subacute stroke: a randomized controlled trial. Arch Phys Med Rehabil 89:393-398.

Ziemann U (2004) TMS and drugs. Clin Neurophysiol 115:1717-1729.

Ziemann U, Lönnecker S, Steinhoff BJ, Paulus W (1996a) Effects of antiepileptic drugs on motor cortex excitability in humans: a transcranial magnetic stimulation study. Ann Neurol 40:367-378.

Ziemann U, Rothwell JC, Ridding MC (1996b) Interaction between intracortical inhibition and facilitation in human motor cortex. J Physiol 496:873-881.

Ziemann U, Tergau F, Wassermann EM, Wischer S, Hildebrandt J, Paulus W (1998) Demonstration of facilitatory I wave interaction in the human motor cortex by paired transcranial magnetic stimulation. J Physiol 511: 181-190. 